\title{
Ligantes hidráulicos e a hidratação de óxido de magnésio em concretos refratários
}

\section{(Hydraulic binders and magnesium oxide hydration in refractory castables)}

\author{
R. Salomão ${ }^{1,2}$, L. R. M. Bittencourt ${ }^{3}$, V. C. Pandolfelli ${ }^{1}$ \\ ${ }^{1}$ Grupo de Engenharia de Microestrutura de Materiais, Departamento de Engenharia de Materiais, UFSCar \\ Rod. Washington Luiz, km 235, C.P. 676. S. Carlos, SP 135656-905 \\ ${ }^{2}$ Universidade Federal do ABC, R. Catequese 242, Santo André, SP 09090-400 \\ ${ }^{3}$ Magnesita S.A., Praça Louis Ensch 240, Contagem, $M G$ \\ vicpando@power.ufscar.br,rafael.salomao@ufabc.edu.br
}

\begin{abstract}
Resumo
A presença de cimento de aluminato de cálcio e alumina hidratável pode afetar significativamente o comportamento de hidratação do óxido de magnésio $(\mathrm{MgO})$ em suspensões aquosas. Entre outros efeitos, destaca-se a formação do composto conhecido como hidrotalcita $\left(\mathrm{Mg}_{6} \mathrm{Al}_{2}\left(\mathrm{CO}_{3}\right)(\mathrm{OH})_{16} \cdot\left(4 \mathrm{H}_{2} \mathrm{O}\right)\right)$, que pode potencializar ou inibir os danos causados pela hidratação do $\mathrm{MgO}$, dependendo da quantidade em que é gerado. Este trabalho investigou como a presença desses ligantes afeta a hidratação do $\mathrm{MgO}$ e suas conseqüências em formulações de concretos refratários usando testes de hidratação-desidratação, medidas de pH, expansão volumétrica aparente, resistência à tração por compressão diametral e porosidade. Por meio de comparações com composições de referência sem-ligante e sem-ligante-e-sem- $\mathrm{MgO}$, foi verificado que os efeitos danosos da hidratação do $\mathrm{MgO}$ podem ser significativamente reduzidos por meio do ajuste do tipo e do teor de ligante aos concretos.

Palavras-chave: concretos refratários, cimento de aluminato de cálcio, alumina hidratável, óxido de magnésio, técnicas antihidratação.
\end{abstract}

\begin{abstract}
The presence of calcium aluminate cement and hydratable alumina can significantly modify the magnesia sinter hydration behavior in aqueous suspensions. As a consequence of these studies, the present paper investigates how these binders content could affect magnesia hydration in refractory castables using hydration-dehydration tests, $p H$, AVE, mechanical strength and porosity measurements. Besides this, because for these materials the aspects related to mechanical strength, porosity and refractoriness also must be taken into account, binder-free, magnesia-free and magnesia-and-binder-free samples were also tested as references. It was found out that the deleterious effects of magnesia hydration can be greatly minimized by the binder content.
\end{abstract}

Keywords: Refractory castables, calcium aluminate cement, hydratable alumina, magnesia hydration, anti-hydration technique.

\section{INTRODUÇÃO}

Concretos refratários contendo sínter de $\mathrm{MgO}$ apresentam importantes atrativos tecnológicos como elevada refratariedade e resistência a corrosão por escórias alcalinas e ao dano por choque térmico, devido à formação de espinélio $\left(\mathrm{MgAl}_{2} \mathrm{O}_{4}\right)[1]$. No entanto, o $\mathrm{MgO}$ também reage facilmente com água, gerando hidróxido de magnésio $\left(\mathrm{Mg}(\mathrm{OH})_{2}\right)$ ou brucita [2-5]. Se essa reação ocorrer antes do enrijecimento do concreto (durante o período de estocagem do sínter ou durante a mistura com água), ela pode gerar porosidade após a decomposição da brucita, durante o primeiro aquecimento (400-600 ${ }^{\circ} \mathrm{C}$ ), reduzindo a resistência mecânica do material [5]. Por outro lado, se ocorrer durante a cura e secagem, a expansão volumétrica de até 3 vezes que acompanha a reação de hidratação pode gerar tensões mecânicas e danos mecânicos à estrutura, conhecidos como expansão volumétrica aparente (EVA) [4, 6]. A literatura sugere que, com o uso de técnicas anti-hidratação para o $\mathrm{MgO}$ (TAHM), esse efeito colateral pode ser reduzido e os benefícios da adição de $\mathrm{MgO}$ maximizados [5-7]. Entre aquelas melhor descritas, o controle do teor e tipo de ligante utilizado [8, 9] destaca-se como uma das mais eficazes. O cimento de aluminato de cálcio $(\mathrm{CAC})$ e as aluminas hidratáveis $(\mathrm{AH})$ são os ligantes hidráulicos mais utilizados em formulações de concretos refratários, garantindo resistência mecânica adequada para desmoldagem, até a sinterização do material. Essa técnica está relacionada às reações que ocorrem quando eles se hidratam em presença de $\mathrm{MgO}$. O CAC inicialmente se dissolve em água, liberando íons $\mathrm{CA}^{2+}, \mathrm{Al}^{3+}$ e $\mathrm{OH}$. Após um período de saturação, esses íons se combinam para formar hidratos de aluminato de cálcio (como $\mathrm{CaO} \cdot \mathrm{Al}_{2} \mathrm{O}_{3} \cdot 10 \mathrm{H}_{2} \mathrm{O}$, $\mathrm{CAH}_{10}$, ou $3 \mathrm{CaO} \cdot \mathrm{Al}_{2} \mathrm{O}_{3} \cdot 6 \mathrm{H}_{2} \mathrm{O}, \mathrm{C}_{3} \mathrm{AH}_{6}$ ), que se precipitam, restringindo o movimento das partículas e enrijecendo a estrutura [10-12]. Durante esse processo, um significativo aumento no $\mathrm{pH}$ do concreto é observado (equações $\mathrm{A}$ e 
B) $[11,12]$. Pelo princípio do deslocamento de equilíbrio químico, o excesso de íons $\mathrm{OH}^{-}$favorece a hidratação do $\mathrm{MgO}$, como mostrado na Equação C [2, 3]:

$$
\begin{aligned}
& \mathrm{Ca}\left(\mathrm{AlO}_{2}\right)_{2}+4 \mathrm{H}_{2} \mathrm{O} \rightarrow \mathrm{Ca}^{2+}+2 \mathrm{Al}(\mathrm{OH})_{4}^{-} \\
& \mathrm{Al}(\mathrm{OH})_{4}^{-} \rightarrow \mathrm{Al}^{3+}+4 \mathrm{OH}^{-} \\
& \mathrm{MgO}+\mathrm{H}_{2} \mathrm{O} \rightarrow \mathrm{MgOH}^{+}+\mathrm{OH}^{-} \rightarrow \mathrm{Mg}(\mathrm{OH})_{2}
\end{aligned}
$$

Testes realizados em suspensões aquosas de CAC e sínter de $\mathrm{MgO}$ mostraram que quanto maior o teor de $\mathrm{CAC}$ na formulação, maior o aumento de $\mathrm{pH}$ e a quantidade de brucita formada, para uma mesma condição de tempotemperatura (7 dias, a $50{ }^{\circ} \mathrm{C}$, em ambiente úmido) [8]. Conseqüentemente, para composições com reduzido teor de CAC, menores níveis de EVA foram observados.

A alumina hidratável é composta por uma fase metaestável e de alta área superficial (acima de $100 \mathrm{~m}^{2} / \mathrm{g}$ ) do óxido de alumínio, conhecida como alumina- $\rho$. Em contato com água, ela se solubiliza parcialmente, gerando um gel de boemita $(\mathrm{AlOOH})$ e pseudo-boemita. Esse gel reduz drasticamente a porosidade da estrutura e após 1 ou $2 \mathrm{~h}$ (acima de $20^{\circ} \mathrm{C}$ ) ele se cristaliza como baierita $(\mathrm{AlOH})_{3}$ ), ligando as partículas $[13,14]$. Quando testada nas mesmas condições que o CAC, diferentes comportamentos foram observados [9, $15,16]$. Acima de um certo teor de AH (5\%peso), nenhuma evidência de hidratação do $\mathrm{MgO}$ foi detectada. Nesse caso, dois efeitos contribuíram para interromper essa reação: 1) a hidratação da $\mathrm{AH}$ afeta menos significativamente o $\mathrm{pH}$ da suspensão 2) a liberação de íons $\mathrm{Al}^{3+}$ permite a formação de uma camada insolúvel e protetora de hidrotalcita $\left(\mathrm{Mg}_{6} \mathrm{Al}_{3}(\mathrm{OH})_{16}\left(\mathrm{CO}_{3} \times 4 \mathrm{H}_{2} \mathrm{O}\right) \quad[17,18]\right.$ na superfície das partículas de $\mathrm{MgO}$, reduzindo a velocidade de hidratação [9]. Com base nessas investigações [8,9], o presente trabalho teve como objetivo verificar a eficácia do controle do tipo e teor de ligante como técnica anti-hidratação para o $\mathrm{MgO}$ em formulações de concreto refratário. Uma composição de concreto contendo sínter de $\mathrm{MgO}$ e diferentes teores de $\mathrm{CAC}$ ou $\mathrm{AH}$ foi avaliada por meio de testes de hidratação, de expansão volumétrica aparente e termogravimetria. Além disso, como nesses materiais a variação do teor de ligante pode afetar significativamente suas propriedades mecânicas, porosidade e refratariedade, composições de referência sem $\mathrm{MgO}$ também foram testadas.

\section{MATERIAIS E MÉTODOS}

Uma composição de concreto refratário contendo 6\%peso de sínter de $\mathrm{MgO}$ (Magnesita S.A., Brasil), $18 \%$ vol. de água (em relação à massa seca de concreto) e diferentes quantidades de cimento de aluminato de cálcio (CA14M, Almatis, EUA, 0-12\%peso) ou alumina hidratável (Alphabond 300, Almatis, EUA, 0-6\%peso) foi empregada nos testes (a AH só foi adicionada até teores de 6\%peso devido ao elevado consumo de água que ocasiona na mistura). Amostras de referência onde o $\mathrm{MgO}$ foi substituído por alumina calcinada (AC) com granulometria equivalente também foram testadas nas mesmas condições. Detalhes das composições testadas podem ser encontrados na Tabela I.

A adição de água e a mistura foram realizadas em um misturador planetário, durante $10 \mathrm{~min}$. As formulações

\begin{tabular}{|c|c|c|c|c|c|}
\hline \multirow[b]{2}{*}{ Componentes } & \multirow{2}{*}{\multicolumn{3}{|c|}{ Matérias primas }} & \multicolumn{2}{|c|}{ \%peso } \\
\hline & & & & $\begin{array}{c}\text { Contendo } \\
\text { ligante e MoO }\end{array}$ & $\begin{array}{l}\text { Com ligante } \\
\text { e sem } \operatorname{MoO} 0 *\end{array}$ \\
\hline \multirow{10}{*}{$\begin{array}{c}\text { Matriz } \\
\left(\mathrm{D}_{\mathrm{P}}<\mathbf{1 0 0} \mu \mathrm{m}\right)\end{array}$} & \multirow{5}{*}{ 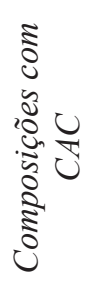 } & \multicolumn{2}{|c|}{ Alumina calcinada (A1000SG, EP 1000)a } & $15-3$ & $21-9$ \\
\hline & & \multicolumn{2}{|c|}{ Cimento de aluminato de cálcio $(\mathrm{CA} 14 \mathrm{M})^{\mathrm{a}}$} & $0-12$ & $0-12$ \\
\hline & & \multicolumn{2}{|c|}{$\mathrm{MgO}\left(\mathrm{D}_{50}=15 \mu \mathrm{m}, 98 \% \text { peso } \mathrm{MgO}\right)^{\mathrm{b}}$} & 6 & 0 \\
\hline & & \multirow{2}{*}{ Aditivos } & Água & $\begin{array}{c}5,3-5,5 \\
{[18 \% \text { volume }]}\end{array}$ & $\begin{array}{c}5,5 \\
{[18 \% \mathrm{vol} .]}\end{array}$ \\
\hline & & & Dispersante (Poli(etileno glicol) ${ }^{\mathrm{d}}$ & 0,2 & 0,2 \\
\hline & \multirow{5}{*}{ 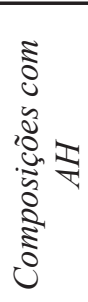 } & \multicolumn{2}{|c|}{ Alumina calcinada (A1000SG, EP 1000) } & $15-9$ & $21-15$ \\
\hline & & \multicolumn{2}{|c|}{ Alumina hidratável (Alphabond 300)a } & $0-6$ & $0-6$ \\
\hline & & \multicolumn{2}{|c|}{$\mathrm{MgO}\left(\mathrm{D}_{50}=15 \mu \mathrm{m}, 98 \% \text { peso } \mathrm{MgO}\right)^{\mathrm{b}}$} & 6 & 0 \\
\hline & & \multirow{2}{*}{ Aditivos } & Água & $\begin{array}{c}5,5-6 \\
{[18 \% \text { volume }]}\end{array}$ & $\begin{array}{c}5,5-5,7 \\
{[18 \% \text { vol. }]}\end{array}$ \\
\hline & & & Dispersante (polietieno glicol) ${ }^{\mathrm{d}}$ & 0,4 & 0,2 \\
\hline $\begin{array}{c}\text { Agregados } \\
\left(\mathrm{D}_{\mathrm{P}} \geq \mathbf{1 0 0} \mu \mathrm{m}\right)\end{array}$ & \multicolumn{3}{|c|}{ Alumina eletrofundida branca ${ }^{c}$} & 79 & 79 \\
\hline
\end{tabular}

Tabela I - Composições de concreto refratário utilizadas.

[Table I - Refractory castables compositions.]

${ }^{a}$ Almatis (EUA); ${ }^{b}$ Magnesita S.A. (Brasil); ${ }^{c}$ Elfusa (Brasil); ${ }^{d}$ Bayer (Alemanha); * Composições de referência 
foram moldadas sob vibração na forma de cilindros de 40 $\mathrm{mm} \times 40 \mathrm{~mm}$, para os testes de secagem e hidratação e medidas de resistência à tração por compressão diametral e porosidade, e $70 \mathrm{~mm}$ x $70 \mathrm{~mm}$, para a avaliação da expansão volumétrica aparente. O período inicial de cura foi realizado em uma câmara climatizada Vöetsch 2020 a $8{ }^{\circ} \mathrm{C}$ durante $24 \mathrm{~h}$. Essas condições foram aplicadas para assegurar um valor mínimo de resistência mecânica dos concretos para desmoldagem e, ao mesmo tempo, que não houvesse hidratação significativa do $\mathrm{MgO}$ [4]. O teste de hidratação consistiu em manter as amostras a $50{ }^{\circ} \mathrm{C}$ em ambiente úmido durante 7 dias. Durante esse período, a resistência à tração por compressão diametral, a porosidade, o perfil de desidratação e a expansão volumétrica aparente foram avaliados a cada $24 \mathrm{~h}$. Em paralelo, logo após a mistura, o $\mathrm{pH}$ dos concretos foi monitorado a cada $10 \mathrm{~s}$ durante $24 \mathrm{~h} \mathrm{em}$ temperatura ambiente $\left(25^{\circ} \mathrm{C}\right)$.

Os testes de desidratação foram conduzidos até $600{ }^{\circ} \mathrm{C}$ em amostras verdes, com taxa de aquecimento $10{ }^{\circ} \mathrm{C} / \mathrm{min}$ e um aparato termogravimétrico [19]. Variações de massa e no perfil de temperatura no interior do forno e na superfície das amostras foram simultaneamente registradas. A taxa de perda de massa percentual (dW/dt, \%/min) em uma certa temperatura foi utilizada na avaliação do comportamento de desidratação $[4,6,19]$.

O teste de EVA consiste em medir as dimensões de amostras cilíndricas de 70 x $70 \mathrm{~mm}$, exposta à umidade, durante 7 dias, em uma dada temperatura. A EVA é calculada considerando-se o volume inicial das amostras como referência e pode ser descrito pelas seguintes equações:

$$
\begin{aligned}
& \mathrm{V}_{\mathrm{i}}=\mathrm{H}_{\mathrm{i}} \times\left(\mathrm{D}_{\mathrm{i}}-2 \mathrm{t}\right)^{2} / 4 \\
& \mathrm{EVA}=100 \times\left(\mathrm{V}_{\mathrm{E}}-\mathrm{V}_{0}\right) / \mathrm{V}_{0}
\end{aligned}
$$

A expressão D é usada para calcular o volume das amostras em um tempo particular, onde $\mathrm{V}_{\mathrm{i}}$ é o volume, $\mathrm{H}$ é a altura, $\mathrm{D}$ é o diâmetro e $\mathrm{t}$ a espessura da parede do molde. Para o parâmetro EVA, $V_{0}$ é o volume inicial da amostra e $V_{E}$ o correspondente após a hidratação e expansão. Uma descrição detalhada da técnica e sua utilização pode ser encontrada na literatura $[4,6,8,9]$.

As medidas de resistência à tração por compressão diametral foram realizadas por meio de compressão diametral, de acordo com a norma ASTM C496-96, em um equipamento MTS TestStar II, com taxa de aplicação de carga constante de $42 \mathrm{~N} / \mathrm{s}$. A porosidade das amostras foi medida utilizando-se o método de imersão em querosene. Ambas técnicas usaram amostras previamente secas a $110^{\circ} \mathrm{C}$ durante $24 \mathrm{~h}$.

\section{RESULTADOS E DISCUSSÃO}

Concretos contendo sínter de $\mathrm{MgO}$ e cimento de aluminato de cálcio

A termogravimetria tem sido utilizada em diversos
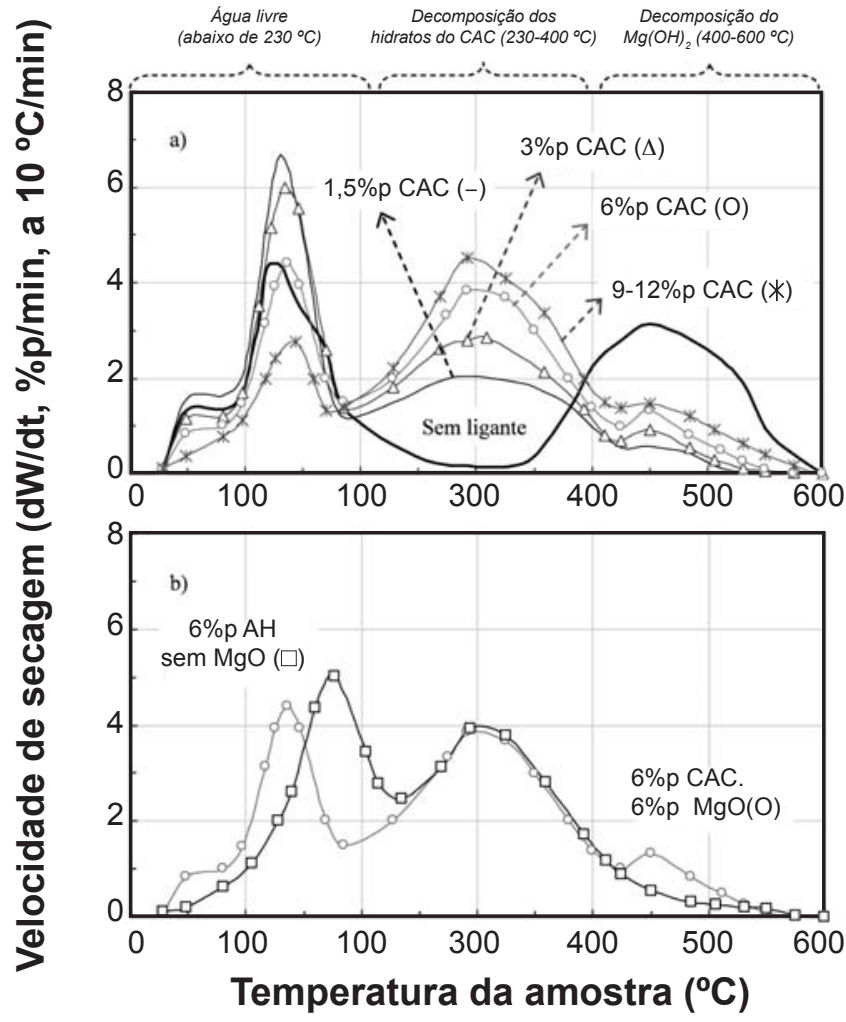

Figura 1: Termogravimetria dos concretos contendo a) 6\%peso de sínter de $\mathrm{MgO}$ e diferentes teores de CAC e b) $6 \%$ peso de CAC e sem $\mathrm{MgO}$ (após 7 dias a $50{ }^{\circ} \mathrm{C}$ em ambiente úmido).

[Figure 1: Thermogravimetry of castables containing a) 6 wt.\% of $\mathrm{MgO}$ sinter and different amounts of calcium aluminate cement and b) $6 \mathrm{wt} \%$ of calcium aluminate cement and no $\mathrm{MgO}$ (after 7 days at $50^{\circ} \mathrm{C}$ in humid environment).]

trabalhos para acompanhar a hidratação/desidratação do $\mathrm{MgO}$ em suspensões e concretos [4, 5, 8, 9]. As curvas de perda de massa em função da temperatura para sistemas contendo $\mathrm{CAC}$ e $\mathrm{MgO}$ geralmente são compostas por 4 picos principais [4]. Aqueles que ocorrem abaixo de $230{ }^{\circ} \mathrm{C}$ representam a saída da água não-quimicamente ligada ou água livre [19]. Os verificados em temperaturas superiores estão relacionados à decomposição dos compostos hidratados do CAC $\left(230-400{ }^{\circ} \mathrm{C}\right)[4,19]$ e brucita $(400-$ $\left.600{ }^{\circ} \mathrm{C}\right)$ [2-4]. As áreas sob esses picos são proporcionais à massa de água liberada nesses intervalos de temperatura $\mathrm{e}$, conseqüentemente, à quantidade de hidratos do CAC e brucita gerados durante o período de cura. A Fig. 1 mostra a termogravimetria das amostras de concretos contendo diferentes teores de CAC após 7 dias a $50{ }^{\circ} \mathrm{C}$ em ambiente úmido.

Pode-se observar que, com o aumento do teor de CAC, mais hidratos $\left(3 \mathrm{CaO} \times \mathrm{Al}_{2} \mathrm{O}_{3} \times 6 \mathrm{H}_{2} \mathrm{O}\right.$ e $\mathrm{Al}(\mathrm{OH})_{3}$, neste caso [12, $20,21])$ e brucita foram formados, reduzindo a quantidade de água livre nas amostras (a amostra sem ligante será analisada nas próximas seções). Como todas as amostras continham uma mesma quantidade de sínter de $\mathrm{MgO}$, esses resultados indicam que, devido ao aumento da quantidade de CAC, a velocidade de hidratação do $\mathrm{MgO}$ foi significativamente 

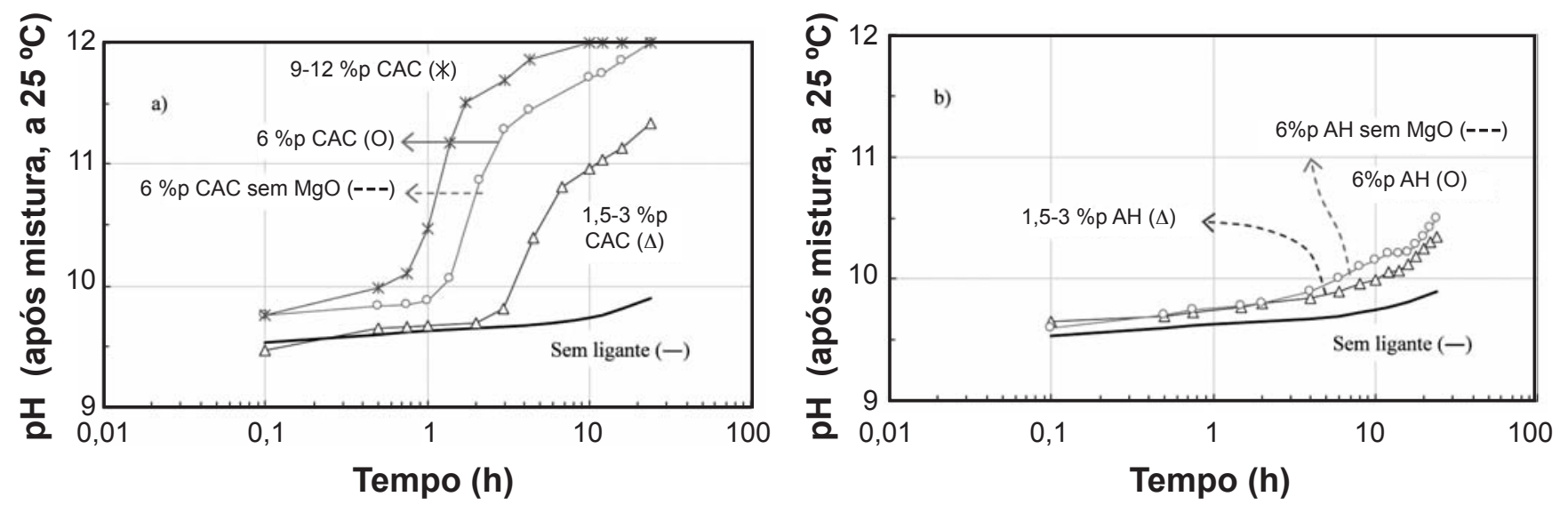

Figura 2: Variação do $\mathrm{pH}$ dos concretos contendo sínter de $\mathrm{MgO}$ preparados com diferentes teores de a) CAC ou b) $\mathrm{AH}$ (as linhas pontilhadas representam os dados referentes às amostras de referência sem $\mathrm{MgO}$ ).

[Figure 2: pH values for castables containing $\mathrm{MgO}$ sinter and different amounts of a) calcium aluminate cement or b) hydratable alumina (the dotted lines highlight the results obtained for the $\mathrm{MgO}$-free reference compositions).]
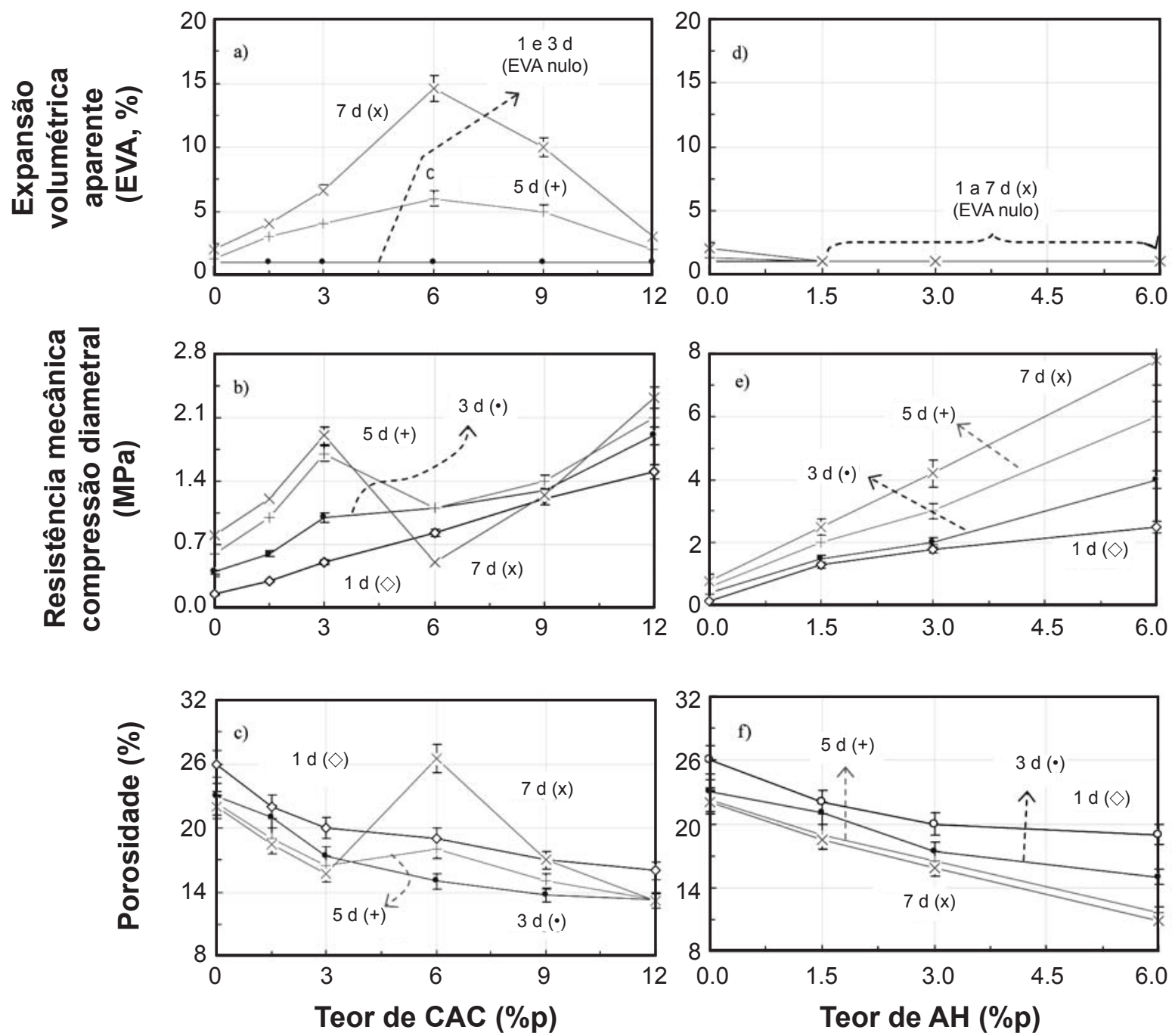

Figura 3: Efeito do teor de CAC ou AH na expansão volumétrica aparente (a, d), resistência mecânica (b, e) e porosidade (c, f) (após 7 dias a $50{ }^{\circ} \mathrm{C}$, em ambiente úmido).

[Figure 3: Effect of the amount of calcium aluminate cement or hydratable alumina on the apparent volumetric expansion (a, d), mechanical strength (b, e) and porosity (c, $f$ ) (during 7 days, at $50{ }^{\circ} \mathrm{C}$, in humid environment).] 
aumentada. Esse comportamento pode ser associado à evolução do pH dos concretos após a mistura (Fig. 2).

Pode ser observado na Fig. 2a que todas as composições com CAC (inclusive as referências sem $\mathrm{MgO}$ ) apresentaram um valor inicial de $\mathrm{pH}$ da ordem de 9,7. Após um período de indução inicial o pH começou a aumentar, estabilizando em valores significativamente superiores. Quanto maior o teor de cimento, mais rapidamente o aumento de $\mathrm{pH}$ ocorreu e maior o nível atingido após $24 \mathrm{~h}$. Resultados similares podem ser encontrados na literatura, associados ao comportamento de dissolução dos íons $\mathrm{Ca}^{2+}, \mathrm{Al}^{3+} \mathrm{e} \mathrm{OH}^{-}$ na água $[11,12]$. Como valores de $\mathrm{pH}$ elevados aumentam a força motriz para hidratação do $\mathrm{MgO}$, os resultados das Figs. 1a e 2a podem ser relacionados, indicando que, também em concretos refratários, a presença de grande quantidade de CAC pode aumentar a velocidade de formação da brucita. No entanto, em concretos refratários, o teor de brucita gerado não pode ser visto como a única variável que contribui para o desenvolvimento da EVA. A hidratação do CAC começa após algumas horas após a mistura, enquanto a do $\mathrm{MgO}$, pode levar alguns dias, dependendo das condições de cura [4]. Essa diferença de velocidade de hidratação pode causar 2 efeitos importantes: 1) A hidratação do CAC e o correspondente aumento de $\mathrm{pH}$ não foram afetados significativamente pela presença do $\mathrm{MgO}$ (Figs. 2a e 2b, linhas pontilhadas); 2) A rápida redução de porosidade e a conseqüente consolidação da estrutura dos concretos diminuíram o espaço disponível para acomodar a brucita gerada. Devido a isso, estabelece-se uma competição entre o volume extra gerado na hidratação do $\mathrm{MgO}$ e a redução de porosidade e o aumento de resistência mecânica necessária para prevenir os danos à estrutura. Nas Figs. 3a, 3b, 3c e $4 \mathrm{a}$, respectivamente, os resultados dessa competição são avaliados considerando a evolução da EVA, resistência à tração por compressão diametral e porosidade para amostras de concreto contendo $\mathrm{MgO}$ e diferentes teores de CAC (0$12 \%$ peso), em função do tempo de cura (até 7 dias, a $50^{\circ} \mathrm{C}$, em ambiente úmido).

Para as amostras contendo 1,5-3 e 12\% peso de CAC, podem ser observados menores valores de EVA e aumentos de resistência à tração por compressão diametral e redução de porosidade com o tempo de cura; para aquelas com 6 e $9 \%$ peso, por outro lado, observou-se uma significativa deterioração das propriedades após o quarto dia de exposição. Nas amostras sem $\mathrm{MgO}$ (Fig. 4a), verificou-se que quanto maior o teor de $\mathrm{CAC}$ maior a resistência à tração por compressão diametral, sem que aumento de porosidade e EVA fossem verificados. Para as amostras com baixo teor de CAC (1,5-3\%peso), os reduzidos níveis de EVA podem ser explicados considerando-se que, apesar da baixa resistência obtida, a pequena quantidade de brucita gerada pôde ser facilmente acomodada na porosidade da estrutura. Aquela com o maior teor de CAC (12\%peso) apresentou elevado grau de hidratação do $\mathrm{MgO}$ e redução de porosidade, no entanto, apesar disso, os valores de EVA observados foram os menores entre as amostras com CAC, devido à elevada resistência alcançada. Em cada um desses casos, uma

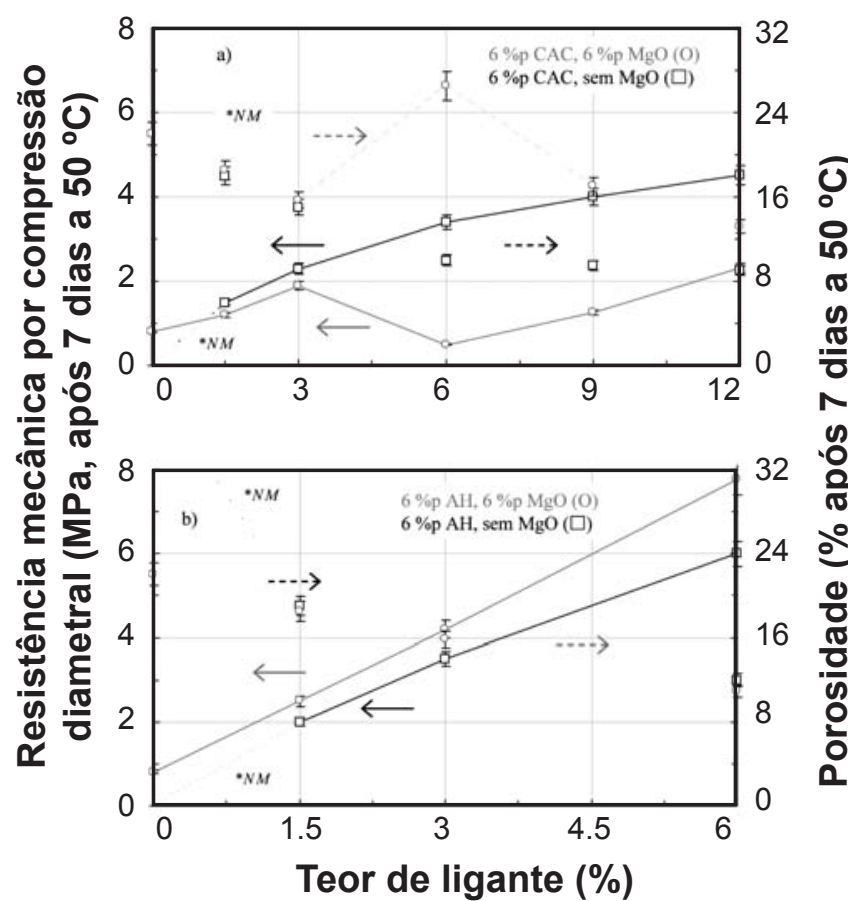

Figura 4: Resistência mecânica e porosidade dos concretos sem $\mathrm{MgO}$ contendo 6\%peso de a) CAC ou b) $\mathrm{AH}$ (após 7 dias a $50{ }^{\circ} \mathrm{C}$, em ambiente úmido) ( ${ }^{*} \mathrm{NM}=$ não mensurável). [Figure 4: Mechanical strength and porosity of $\mathrm{MgO}$-free castables containing $6 \mathrm{wt}$ \% of a) calcium aluminate cement or b) hydratable alumina (after 7 days, at $50^{\circ} \mathrm{C}$, in humid environment) (*NM= measurement not possible).]

das variáveis que contribuem para minimizar a EVA foi otimizada (respectivamente, menor teor de brucita gerada e elevada resistência à tração por compressão diametral mecânica). Para as amostras com teores médios de CAC (6-9\%peso), por outro lado, a combinação de pH alcalino, elevado grau de hidratação do $\mathrm{MgO}$, redução significativa de porosidade nos primeiros dias e níveis intermediários de resistência à tração por compressão diametral favoreceu a EVA e a deterioração da estrutura. Esses resultados indicam que o controle do teor de $\mathrm{CAC}$ nas formulações de concreto pode reduzir, porém não eliminar totalmente, os efeitos deletérios da hidratação do $\mathrm{MgO}$. No entanto, os benefícios do uso de baixos (abaixo de 6\%peso) ou elevados (acima de $9 \%$ peso) teores de CAC devem ser cuidadosamente considerados devido aos efeitos colaterais que podem introduzir. Os concretos ultra-baixo teor de cimento (UBTC) são bastante sensíveis à variações na etapa de cura e aquecimento inicial devido à baixa resistência mecânica desenvolvida [19-22] e requerem a adição de aditivos de secagem, como fibras poliméricas, para reduzir os riscos de explosão [23]. As formulações com alto teor de cimento, por outro lado, podem ter sua refratariedade reduzida devido ao teor excessivo de $\mathrm{CaO}$ [1]. Com base nessas considerações, é razoável supor que o controle do teor de CAC poderia gerar melhores resultados se fosse aplicada em combinação com outras técnicas anti-hidratação, como por exemplo, a adição de agentes quelantes [6]. 


\section{Concretos contento sínter de $\mathrm{MgO}$ e alumina hidratável}

O comportamento de secagem dos concretos ligados com AH difere significativamente daqueles contendo CAC [23, 24]. Devido aos baixos níveis de porosidade e permeabilidade da estrutura, durante a secagem dos concretos com $\mathrm{AH}$ a liberação de água livre e a decomposição dos compostos hidratados formados se fundem em um único estágio (entre a temperatura ambiente até por volta de $300^{\circ} \mathrm{C}$ ); entre 300$500{ }^{\circ} \mathrm{C}$, a baierita se decompõe [24]. Considerando esses aspectos, pode-se observar na Fig. 5a que houve diferenças pouco significativas entre os resultados de termogravimetria para as amostras com diferentes teores de $\mathrm{AH}$ (1,5-6\%peso, Fig. 5a), relacionadas com as reações de decomposição que ocorrem abaixo de $300{ }^{\circ} \mathrm{C}$. A comparação com a amostra de referência (Fig. 5b) mostra que não houve hidratação significativa do $\mathrm{MgO}$ e que essas mudanças foram introduzidas principalmente pela variação do teor de $\mathrm{AH}$ (devido à geração de grande quantidade de boemita e redução de permeabilidade).

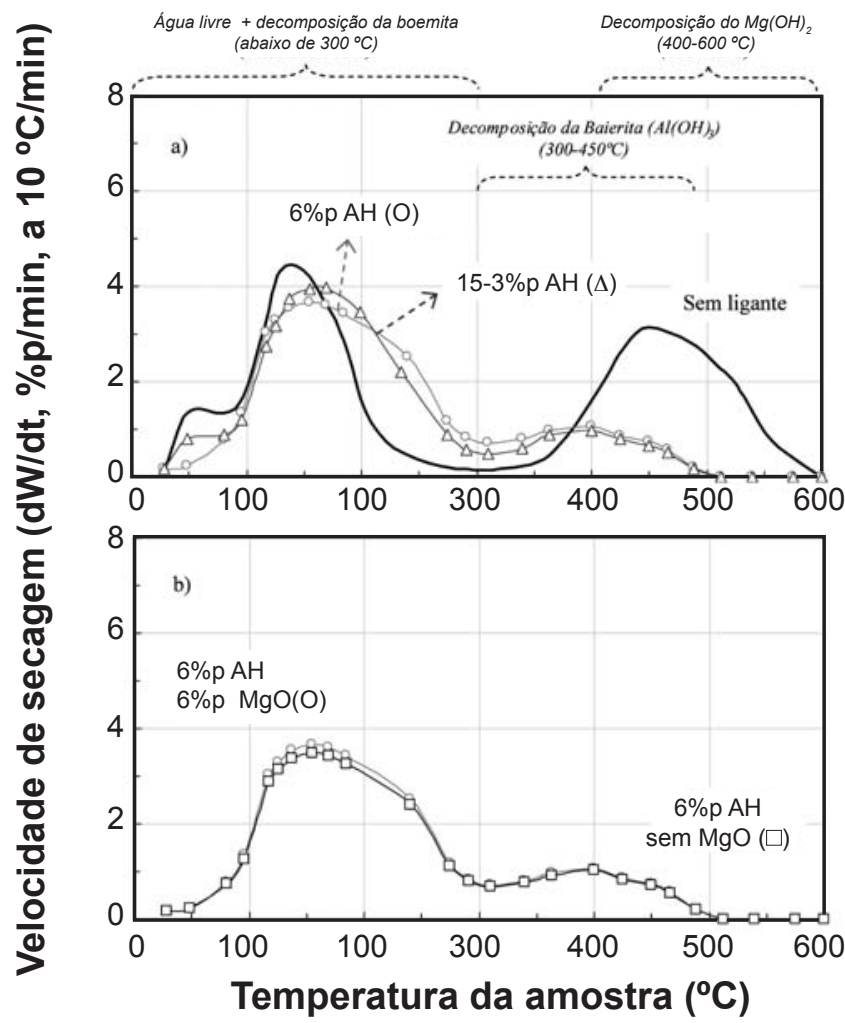

Figura 5: Termogravimetria de concretos contendo a) 6\%peso de sínter de $\mathrm{MgO}$ e diferentes teores de $\mathrm{AH}$ e b) 6\%peso de $\mathrm{AH}$ sem $\mathrm{MgO}$ (após 7 dias a $50{ }^{\circ} \mathrm{C}$, em ambiente úmido).

[Figure 5: Thermogravimetry of castables containing a) 6 wt.\% of $\mathrm{MgO}$ sinter and different amounts of hydratable alumina and b) 6 wt.\% of hydratable alumina and no $\mathrm{MgO}$ (after 7 days, at $50{ }^{\circ} \mathrm{C}$, in humid environment)]

Ao mesmo tempo em que nenhuma EVA significativa foi observada (Fig. 3d), foram obtidos níveis de resistência à tração por compressão diametral (Fig. 3e) até 2,5 vezes superiores e com menores porosidades (Fig. 3f) que a composição equivalente com $\mathrm{CAC}$ e $25 \%$ mais resistente que a amostra de referência sem $\mathrm{MgO}$ (Fig. 4b). Esses pontos indicam que, por meio da substituição do $\mathrm{CAC}$ por $\mathrm{AH}$, não apenas a hidratação do $\mathrm{MgO}$ e seus efeitos podem ser minimizados, mas interessantes benefícios foram obtidos em relação às propriedades mecânicas do concreto, como registrado em outros trabalhos [25]. Esse comportamento pode ser atribuído a dois efeitos principais. Inicialmente, como visto na Fig. 2b, pode ser observado que a adição de $\mathrm{AH}$ não alterou o $\mathrm{pH}$ dos concretos tão significativamente quanto o $\mathrm{CAC}$, reduzindo assim a força motriz para hidratação do $\mathrm{MgO}$. Além disso, a combinação de sínter de $\mathrm{MgO}$ e $\mathrm{AH}$ produz uma pequena quantidade de hidrotalcita (HTC). A HTC é formada quando a razão de íons $\mathrm{Mg}^{2+}$ e $\mathrm{Al}^{3+}$ está próxima de 3:1 (outras relações geram HTC mais boemita ou brucita [17, 18]) e, devido a isso, se forma preferencialmente na superfície das partículas de $\mathrm{MgO}[9,16,25]$. Devido à sua baixa solubilidade em ambiente alcalino e à presença de ligações tipo pontes de hidrogênio, ela atua como camada protetora na superfície do $\mathrm{MgO}$ ao mesmo tempo que liga fortemente as partículas da matriz. Uma vez formada, não há mais íons $\mathrm{Mg}^{2+}$ disponível na suspensão e a geração de HTC é interrompida. Como a quantidade de HTC gerada nessas condições está abaixo do limite detectável pelo equipamento de termogravimetria empregado (na literatura, DRX qualitativa foi utilizada para esse fim [9]), nenhuma diferença significativa foi observada no comportamento de secagem dos concretos. Comparando com as composições com $\mathrm{CAC}$, o controle do teor de AH gera importantes benefícios, com pequenos efeitos colaterais. 1) Para os maiores teores de $\mathrm{AH}$, quantidades extras de água e dispersantes (de 5,5 para $6 \%$ peso e 0,2 para $0,4 \%$ peso, respectivamente) tiveram que ser adicionados à formulação para melhorar a fluidez do concreto. 2) Os conhecidos problemas relacionados à explosões durante os estágios iniciais do aquecimento de concretos ligados com $\mathrm{AH}$ podem ser resolvidos utilizado-se fibras poliméricas como aditivos de secagem [23, 24]. No entanto, a ausência de $\mathrm{CaO}$ em sua composição pode favorecer significativamente a refratariedade do material [25].

\section{Concretos contendo $\mathrm{MgO}$ sem ligante}

A composição sem ligante apresentou um comportamen-to inesperado e potencialmente útil. Observando-se os re-sultados apresentados nas Figs. 1, 3 e 4, pode-se notar que, apesar do elevado grau de hidratação do $\mathrm{MgO}$ (mais elevado entre todas as amostras testadas), a amostra sem ligante apresentou baixos valores de EVA e aumento de resistência à tração por compressão diametral e redução de porosidade contínuos com o tempo de cura. Como nenhum ganho de resistência significativo foi observado na amostra sem ligante e sem $\mathrm{MgO}$ (amostras não puderam ser desmoldadas, Fig. 4), é razoável supor que a hidratação do sínter de $\mathrm{MgO}$ tenha gerado algum efeito ligante. Dois pontos importantes podem ser ressaltados: 1) O elevado grau de hidratação do sínter de $\mathrm{MgO}$ apresentado pela amostra sem ligante pode ser associado à grande disponibilidade de água para reação (sem competições com o $\mathrm{CAC}$ ou $\mathrm{AH}$ ) e à ausência de íons $\mathrm{Al}^{3+}$ para forma hidrotalcita; 2) Composições sem ligante (também conhecidas como zero-cimento) geralmente 
apresentam elevada porosidade, como descrito na literatura e detectado na Fig. 3c. Em concretos contendo ligantes como o CAC e a AH, uma porção significativa da porosidade é ocupada pelos hidratos formados, tornando a estrutura desses materiais altamente sensível a deformações. Nesses casos, a expansão volumétrica que segue a hidratação do $\mathrm{MgO}$ não pode ser convenientemente acomodada e as tensões de compressões geram a EVA e danos mecânicos. Na composição sem ligante, por outro lado, o efeito oposto pôde ser observado: existe grande disponibilidade de espaço para acomodar o volume gerado na hidratação do $\mathrm{MgO}$ e a falta de resistência mecânica permite que pequenos movimentos de acomodação interna ocorram sem EVA. Finalmente, o preenchimento dos poros da estrutura com brucita leva a pequenos, porém significativos, ganhos de resistência mecânica. Esses resultados possuem um importante apelo tecnológico, pois sugere que é possível o desenvolvimento de um ligante magnesiano. Esse ponto será explorado mais detalhadamente nas próximas publicações dos autores.

\section{CONCLUSÕES}

A hidratação do $\mathrm{MgO}$ e a expansão volumétrica aparente foram significativamente afetadas pelo tipo e quantidade de ligante empregado na formulação do concreto. Foi observado que a hidratação do $\mathrm{MgO}$ produziu menos danos nas composições com pequeno (1,5-3 \%peso) ou elevado (12\%peso) teor de CAC. No primeiro caso, esse efeito pode ser associado aos menores elevação do $\mathrm{pH}$, geração de brucita e redução de porosidade; no segundo, à elevada resistência à tração por compressão diametral alcançada. As composições com teores intermediários de CAC (6-9 \%peso) apresentaram os maiores níveis de dano devido à uma combinação de elevado $\mathrm{pH}$, baixa porosidade e resistência mecânica intermediária, resultando em elevado grau de hidratação, pouco espaço para acomodar a brucita formada e pouca resistência para evitar a expansão. Devido aos efeitos colaterais associados à utilização de pouco ou muito CAC em formulações de concreto, pode-se supor que o controle do teor de CAC deva ser aplicado em combinação com outras técnicas anti-hidratação produza melhores resultados. A substituição do CAC por AH gerou interessantes benefícios como a interrupção quase que total da reação de hidratação do $\mathrm{MgO}$ e ganhos significativos de resistência à tração por compressão diametral em comparação às formulações com CAC e mesmo em relação às referências ligadas com $\mathrm{AH}$ e sem $\mathrm{MgO}$. Esses benefícios foram atribuídos à formação de uma camada protetora de hidrotalcita na superfície das partículas de $\mathrm{MgO}$ que reduz a velocidade de hidratação e ainda aumenta a força de ligação entre as partículas. Com base nesses resultados e em seu potencial uso na geração de espinélio in situ, pode apontar o uso das AH como ligante como uma das mais eficazes técnicas anti-hidratação para o $\mathrm{MgO}$ já avaliadas.

\section{AGRADECIMENTOS}

Os autores agradecem à FAPESP, Alcoa Alumínio (Brasil),
Magnesita S.A. (Brasil) e Almatis (EUA) pelo suporte fornecido a este trabalho.

\section{REFERÊNCIAS}

[1] A. Nishikawa, "Technology of monolithic refractories," Tech. Rept. N. 33-7, PLIBRICO, Tokyo, Japão (1984) pp. 98101.

[2] G. K. Layden, G. W. Brindley, J. Am. Ceram. Soc. 46, 11 (1963) 518-522.

[3] A. Kitamura, K. Onizuka, K. Tanaka, T. Overseas 16, 3 (1995) 3-11.

[4] R. Salomão, L. R. M. Bittencourt, V. C. Pandolfelli, Ceram. Int. 33, 5 (2007) 803-810.

[5] R. Salomão, V. C. Pandolfelli, Ceram. Int. 34, 8 (2008) 1829.

[6] L. F. Amaral, I. R. Oliveira, R. Salomão, V. C. Pandolfelli, Cerâmica 55, 336 (2008) 400-407.

[7] A. Kaneyasu, S. Yamamoto, A. Yoshida, T. Overseas 17, 2 (1996) 21-26.

[8] R. Salomão, L. F. Amaral, V. C. Pandolfelli, Cerâmica 56, 338 (2008) 134-139.

[9] M. O. C. Villas Boas, R. Salomão, V. C. Pandolfelli, Cerâmica 53, 328 (2007) 361-367.

[10] K. M. Parker, J. H Sharp, Brit. Ceram.Trans. J. 81 (1982) 35-42.

[11] C. M. George, "Aspects of calcium aluminate cement (CAC) hydration", Proc. Refractories Symp., Am. Ceram. Soc. (1994) 1-21.

[12] J. R. Garcia, I. R. de Oliveira, V. C. Pandolfelli, Cerâmica 53 (2007) 42-56.

[13] Y. Hong, Taikabutsu Overseas 9, 1 (1988) 35-38.

[14] W. Ma, P. Brow, J. Am. Ceram. Soc. 82, 2 (1999) 453456.

[15] K. G. Ahari, J. H. Sharp, W. E. Lee, J. Eur. Ceram. Soc. 22 (2002) 495-503.

[16] G. Ye, T. Trocynski, Ceram. Int. 32 (2006) 257-262.

[17] R. Reichle, Chemtech 16 (1986) 58-63.

[18] A. Vaccari, Appl. Clay Sci. 14 (1999) 161-198.

[19] M. D. M. Innocentini, F. A. Cardoso, M. M. Akyioshi, V. C. Pandolfelli, J. Am. Ceram. Soc. 86, 7 (2003) 1146-1148.

[20] P. Garcés, E. G. Alcocel, S. Chindón, C. G. Andreu, J. Alcaide, Cem. Conc. Res. 27, 9 (1997) 1343-1355.

[21] F. A. Cardoso, M. D. M. Innocentini, M. M. Akiyoshi, V. C. Pandolfelli, J. Eur. Ceram. Soc. 24, 7 (2004) 2073-2078.

[22] F. A. Cardoso, M. D. M. Innocentini, M. M. Akiyoshi, V. C. Pandolfelli, Ref. Appl. News 9, 2 (2004) 12-16.

[23] R. Salomão, M. R. Ismael, V. C. Pandolfelli, Ceram. Forum Int. 84 (2007) 103-108.

[24] F. A. Cardoso, M. D. M. Innocentini, M. F. S. Miranda, F. A. O. Valenzuela, V. C. Pandolfelli, J. Eur. Ceram. Soc. 24 (2004) 797-802.

[25] G. Ye, T. Troczynski, J. Mater. Sci. 40 (2005) 3921-3926.

[26] I. R. Oliveira, A. R. Studart, B. Menegazzo, V. C. Pandolfelli, Am. Ceram. Soc. Bull. 81, 12 (2002) 27-34.

(Rec. 17/04/2008, Rev. 23/06/2008, Ac. 26/06/2008) 